\title{
Towards a context aware modeling of trust and access control based on the user behavior and capabilities
}

\author{
Abdallah Mhamed ${ }^{1}$, Meriem Zerkouk ${ }^{2}$, Anas El Husseini ${ }^{1,3}$, \\ Belhadri Messabih², Bachar El Hassan ${ }^{3}$, \\ ${ }^{1}$ Institut Mines-Télécom / Télécom SudParis - CNRS Samovar UMR 5157 Evry - France. \\ abdallah.mhamed@it-sudparis.eu \\ ${ }^{2}$ University of Sciences and Technology Oran, Algeria. \{zerkouk.meriem, bmessabih\}@ gmail.com \\ ${ }^{3}$ Lebanese University, LASTRE Lab. , Tripoli, Lebanon
}

\begin{abstract}
During the last decade, several context based security models has been proposed to take into account the behavioral side of users. However the context parameters studied are mainly related to the device use and the spatio-temporal features, which may led to a weak and inappropriate contextual modeling. By using the huge and various contextual data issued from the sensors, our objective is to provide a security framework suitable for dependant people. This paper shows our approach to model both trust and access control based on the user behavior and capabilities which can be derived from the sensed data.
\end{abstract}

Keywords: Smart environment, trust evaluation, authentication, access control, device usability, user behavior, user capability, context awareness.

\section{Context, motivations and challenges}

\subsection{Security in smart environments}

Pervasive systems contribute significantly to the deployment of personalized services in smart environments. When considering dependant users within their living spaces, security requirements still remain an open issue. While pervasive environments raise new security challenges, they also bring new opportunities owing to ubiquitous technologies and ambient intelligence which provide valuable contextual information about the user and his environment.

Context-aware based security is an emerging approach to deal with the new security problems introduced by the high dynamicity and heterogeneity of mobile devices that characterize pervasive and highly dynamic computing environments. We believe that this can be really achieved owing to context awareness which allow us to benefit from sensing and mobile technologies to derive more accurate contextual data about the user profile and his environment.

Authentication and access control are the pre-requisite security services which are needed to check the identity of users and the resources they can access to. For this reason, our work is focused on these two security issues tightly linked to users. 


\subsection{User Authentication devices}

Usually the adoption of authentication devices relies on three factors [1]: effectiveness, cost and user acceptance. An underlying constraint of most existing authentication techniques is that they require the user to actively do something in order to be authenticated. When these devices are used by physical or mental impaired people, we have to consider two important additional factors:

- Usability: the ease with which people can interact with any device/technology.

- Non Intrusiveness: the use in a discrete and transparent manner without any inconvenience for the user.

The various impacts of several different disabilities on the security and usability of many existing authentication means have been demonstrated in [2]. In some situations the authentication device can be considered as intrusive, and it is therefore desirable to have methods that may be applied transparently, without the need to interrupt with legitimate user activities. By using the anatomy of human head and the dynamics of human voice, the Head Authentication Technique (HAT) proposed in [3] is a non intrusive biometric technique to provide a continuous and transparent authentication.

Despite the huge previous research work, the problem of achieving adequate and effective user authentication still remains an ongoing challenge. Among the range of authentication techniques (based on secret knowledge, token or biometrics), no single method can be considered as applicable to all contexts and for all. Furthermore, the authentication process is mainly based on the provision of some credentials (PIN codes, passwords and even biometric templates) which can be forged or replayed and by the way is exposed to potential spoofing attacks.

Recently, a new approach has been emerged by using the behavioral features of users. A feasibility of having such authentication was studied by EL-Khazzar [4] who proposed an approach based on psychological mechanisms through a 3D graphical maze. A user authentication on mobile devices based on user's behavior and spatio-temporal context was adopted by Rocha [5]. A user behavioral model based on activities, environmental contexts, and user profile is proposed by Lima [6]. Brosso and al. has designed a continuous authentication system using behavioral analysis of users, based on the evidences of behavior to establish trust levels to continuously authenticate the user [7].

\section{Our context aware framework}

A pervasive environment is characterized by a richness of contexts in which users, devices and agents are mobile. The availability of contextual data provided by sensors can be used to extract behavior patterns of the mobile entities (users, devices, agents). Context awareness can bring a valuable help to understand the relation between users, devices and environments. Owing to the big range and variety of sensors deployed, the pervasive space can provide a very rich and valuable information set which can be used to derive: 
- the "dynamic profile" of users (social relationship and user behavior),

- the user capabilities, for aging and people having disabilities.

The objective of our approach is the design and implementation of a context aware framework illustrated in Figure 1.

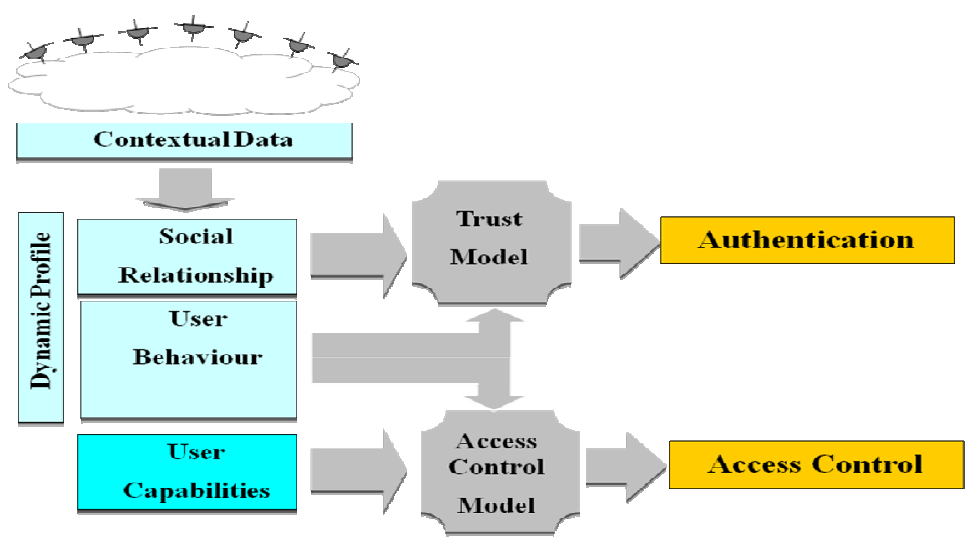

Fig. 1. Our Context Aware framework

By combining the dynamic profile with the user capabilities, our research strategy is motivated by the following challenging tasks:

$1^{\circ}$ ) to identify the user discreetly and transparently by removing the constrained nature of authentication devices

$2^{\circ}$ ) to provide an adaptable service of users based on their capabilities and behavior to ensure more personalization and suitable security.

By the way, the user behavior can be combined with the user capabilities to build a dynamic trust and access control models.

The contextual data collected from sensors are used to derive user behavior patterns which will contribute to set up an implicit and continuous authentication process without using any intrusive device (Figure 2).

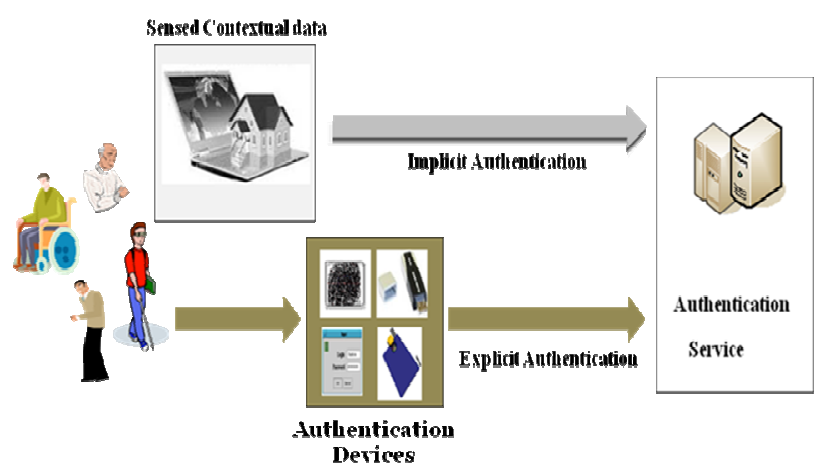

Fig. 2. A transparent authentication process 


\subsection{Contextual trust}

Contextual information open new opportunities for the establishment of trust relations between communicating entities. Trust models based on user abilities, user behavior, user preferences and contextual factors must be explored to improve trust models.

The recent literature brings some interesting ideas going towards the new vision of trust modeling and its contribution to built new context aware security frameworks. To handle the dynamic and variable nature relationships in pervasive environments, some new properties of trust were considered in [8]. Boukerche et al. proposed a novel trust evaluation prototype in which the updating process of trust value is rely on the cooperation level of nodes. Honest behavior will be rewarded while malicious behavior will be penalized [9]. According to Tanveer Zia et al, the recent trust models migrate from a single dimension to multi-dimension trust calculation models by merging the history, the experience and the context of related entities [10]. The given review of trust models show that some basic elements of context such as time and context similarity are considered. However none of the existing works gives a common and unified consideration on all factors that influence the trust.

We have proposed a context-aware trust model based on user behavior by taking into consideration both the user profiles and context attributes [11]. We introduced a protection against the malicious threats affecting the trust evaluation process. We also improved the accuracy of trust metrics based on the right human behavior in situations that require trust.

\subsection{Contextual access control}

For pervasive systems, the access control is handled by the development of context aware based access control models which rely on context data to assign the permission to the users (roles) in the right situation which makes the model dynamic according to the change of context over the time. Extended RBAC models [12] are based on context awareness. Their aim is to improve RBAC by assigning the right access more dynamically. The access is based on the validity of the context by adding to RBAC a single contextual data which is spatial, temporal or environmental $[13,14,15]$. According to our literature review the current access control policies do not take into account the user impairments nor the behavior of users.

We have proposed a context-aware access control model based on user behavior and capabilities [16]. The model is based on ontology learning, enriching and evolution which support continuous learning of behavior and capability patterns. 


\section{Trust Modeling}

The main features of our proposed trust model [11] are given below:

- It combines many of the good features presented in other models, like trust recommendations and the idea of trust distribution, and service-dependent trust;

- It introduces the new concept of Judgment to imitate the human rational thinking on evaluating the ability of recommending entities.

- It deploys a new scheme to detect any abnormal behavior of the nodes,

- It provides different levels of trust based on requested services.

- It provides a user rating that allows taking feedback from users and integrating it in the trust evaluation process.

The three metrics described below are the significant contributions which enhance the process of trust evaluation.

\subsection{Trustworthiness}

The calculation of a trustworthiness value includes the direct trust and the indirect trust. Direct trust is what is commonly called "Risk Assessment". It is used for dealing with newcomers which the entity has not yet any past records of trust evaluation. In case where trust is service-dependent, we added a multiplicative factor to the number of negative actions. This factor is called the Security Action Coefficient $(\boldsymbol{S A C})$. This coefficient refers to the security level of a service.

Direct trust is obtained using:

$$
D T=\frac{\Sigma P A_{i}}{\Sigma P A_{i}+S A C \times \Sigma N A_{i}} .
$$

$\boldsymbol{P} \boldsymbol{A}_{i}$ is the number of positive actions done by the given node and noticed by node $\boldsymbol{i}$. $N \boldsymbol{A}_{i}$ refers to the number of negative actions.

$$
\text { The indirect trust is given by: } \quad I T=\frac{\Sigma T w_{i} \times J_{i}}{n} .
$$

$\boldsymbol{T} \boldsymbol{w}_{\boldsymbol{i}}$ and $\boldsymbol{J}_{\boldsymbol{i}}$ are the trustworthiness and judgment values of the node $\boldsymbol{i}$.

The net trustworthiness is a combination of direct and indirect trust values:

$$
T w=\alpha_{D T} \times D T+\alpha_{I T} \times I T .
$$

where $\boldsymbol{\alpha}_{\boldsymbol{I T}}$ is the indirect trust coefficient given by:

$$
\alpha_{I T}=\frac{T S_{\text {self }}}{T S_{\text {self }}+\Sigma \frac{T S_{i}}{n_{\text {recomm } m}}} \times \frac{\Sigma J_{i}}{n_{\text {tot }}} .
$$

and $\boldsymbol{\alpha}_{\boldsymbol{D T}}$ the direct trust coefficient is given by:

$$
\alpha_{D T}=1-\alpha_{I T} .
$$

$\boldsymbol{T} \boldsymbol{S}_{\text {self }}$ refers to the timestamp of the trust value of the node itself, while $\boldsymbol{T} \boldsymbol{S}_{\boldsymbol{i}}$ denotes the timestamp of the trust value of the node $\boldsymbol{i}$. $\boldsymbol{n}_{\text {tot }}$ is the total number of nodes in 
the network, whereas $\boldsymbol{n}_{\text {recomm }}$ is the number of nodes that responded with recommendations.

\subsection{Judgement}

Judgment is one of the new features introduced that aims to imitate the human behavior in a technical approach. The judgment ability is represented by the overall experience of dealing with the node in question. That experience includes both the total number of control messages exchanged and the total number of actions whether positive or negative. The two judgment values related to the number of actions $\left(\boldsymbol{J}_{\boldsymbol{A}}\right)$ and messages $\left(\boldsymbol{J}_{\boldsymbol{M}}\right)$ exchanged are given below:

$$
J_{A}=\frac{\Sigma A_{i}}{\text { Maximum A }} \quad J_{M}=\frac{\Sigma \text { messages }_{i}}{\text { Maximum messages }_{\text {maxim }}}
$$

At last, the overall judgment value is:

$$
J=J_{A} \times J_{M}
$$

\subsection{User rating}

Since trust is a concept initially borrowed from humans we decided to add a human-based indicator in our model to indicate how much users are satisfied by the quality of service provided in smart environments. Therefore, we have considered the "User rating" as a human recommendation and integrates it in our formula to calculate the final value of trust which depend on the number of users that have rated the service, and how many times they used the service.

\section{User Behavior and Capability based Control Access}

We also proposed a new User Behavior and Capability based Access Control Model [16]. The design of our proposed access control model is an ontologylearning and evolving security policy for predicting the future actions of dependent people. This is reached by reasoning about historical data, contextual data and user behavior according to the access rules that are used in the inference engine to provide the right service according to the user's needs.

The proposed model has the following components: Users $(U)$, Services $(S)$, Devices $(D)$, Environment $(E n v)$ and permission $(P)$, where each entity is described with a set of attributes:

Entity $=\{$ att 1, att $2, \ldots$, attn $\}$.

Policy: $\langle(U, S, D, E n v), P\rangle$

where $P=\{$ permit, deny, obligation or recommendation $\}$.

Authorization decision is assigned according to the combination of all the entities. 
We provide and construct an intelligent security policy specification following the main four steps:

$\left.1^{\circ}\right)$ Behavior tracking: to collect the data about the person, the environment and the activities.

$\left.2^{\circ}\right)$ Profile capability identification: According to the collected data, we define some discriminate factors to distinguish between the different behavior patterns. The next task consists to authenticate the users then to analyze the contextual data for attributing to each user included in behavior classes the right decision.

$\left.3^{\circ}\right)$ Access control policy modeling and reasoning: It consists to represent data on standard format by using ontologies to ensure the interoperability, the sharing and the reuse of security policy. The current captured data and the inference rules are stored in the database to deduce a new knowledge and to check the consistency of the ontology.

$\left.4^{\circ}\right)$ Evolving: A learning process is a continuous monitoring data provided over the time from different sources in order to update the behavior classes.

Our ontology model was built using three case studies: deaf person, blind person, Alzheimer person. We have shown the efficiency of an adaptive access control and the role of tracking the behavior, profile capability in such adaptive access control system. The access control is ensured according to the assignment of the users to behavior and capability groups then we check the valid time, location, device, service and environment to assign the "permit" or "deny" decision.

\section{Conclusion}

The proposed trust model has been implemented using Java language and Eclipse IDE platform. A server in our architecture is the device that provides the service to other nodes. It can be a computer, an RFID reader, or even a sensor. Our model will be tested on Android mobile phones using Android SDK tools in Java. A real evaluation will takes place in a residence dedicated to physically impaired people.

The proposed access control model is based on the semantic web technologies (OWL language, SWRL for rule specification and SPARQL to query the ontology). The ongoing work is aiming to deeply explore the behavior clustering and classification tools and to improve our sensing data base. We are planning to deploy our model in Tele-monitoring health care platform to provide automatic assistance for dependent and frail people living alone.

\section{References}

1. Furnell, S.M., Dowland, P.S., Illingworth, H.M., Reynolds, P.L.: Authentication and Supervision: A survey of User Attitudes. In: J. Computers \& Security. Vol 19, No 6, pp. 529--539 (2000) 
2. Helkala, K.: Disabilities and Authentication Methods: Usability and Security. In: 7th International Conference on Availability, Reliability and Security, pp. 327-334 (2012)

3. Rodwell, P.M., Furnell, S.M., Reynolds, P.L.: A Non-Intrusive Biometric Authentication Mechanism Utilizing Physiological Characteristics of the Human Head. In: J. Computers \& Security. Vol 26, No , pp. 468--478, Elseiver (2007)

4. Al-Khazzar, A., Savage, N.: A2BeST: Graphical Authentication Based on User Behaviour. In: International Conference on , pp. -- (2010)

5. Rocha, C.C., Lima, J.C.D., Dantas, M.A.R., Augustin, I.: A2BeST: An Adaptive Authentication Service Based on Mobile User's Behavior and Spatio-Temporal Context. In: th International Conference on , pp. 771--774. Springer, Heidelberg (2011)

6. Lima, J.C.D., Rocha, C.C., Augustin, I., Dantas, M.A.R.: A Context Aware Recommendation System to Behavioral Based Authentication in Mobile and Pervasive Environments. In: 9th International Conference on Embedded and Ubiquitous Computing, pp. 312--319. (2011)

7. Brosso, I., La Neve, A.: A Continuous Authentication System Based On User Behavior Analysis. In: th International Conference on Availability, Reliability and Security, pp. 380--385. (2010)

8. Ahamed, S. I., Haque, M. M., Hoque, Md. E., Rahman, F., Talukder, N. : Design, Analysis and deployment of omnipresent Formal Trust Model (FTM) with trust bootstrapping for pervasive environments. In: J. of Systems and Software, Vol. 83, No 2, pp. 253--270 (2010)

9. Boukerche, A., Yonglin, R. : A secure mo bile healthcare system using trust-based multicast scheme. In: J. of IEEE on Selected Areas in Communications, Vol. 27, No 4, pp. 387--399 (2009)

10.Zia, T., Islam, M. Z.: Communal Reputation and Individual Trust (CRIT) in Wireless Sensor Networks. In: International Conference on Availability, Reliability and Security, pp. 347--352. (2010)

11.El Husseini A., M'hamed A., El-Hassan B., Mokhtari M.: Trust-based Authentication Scheme with User Rating for Low-ressource Devices in Smart Environments. In: J. Personal and Ubiquitous Computing, Vol. 10, N 1, pp 21--27 (2012)

12.Asmidar. A, Roslan. I., Jamilin, J.: A Review on Extended Role Based Access Control (E-RBAC) Model in Pervasive Computing Environment. In: IEEE International Conference on Intelligent Pervasive Computing, pp. 533--535 (2009)

13.Sadat E. S., Amini M., Zokaei S.: A Context-Aware Access Control Model for Pervasive Computing Environments. In: IEEE International Conference on Intelligent Pervasive Computing, pp.51--56 (2007)

14.Yao. H, Hu. H, Huang B., Li. R.: Dynamic Role and Context Based Access Control for Grid Applications. In: the 6th International Conference on Parallel and Distributed Computing, Applications and Technology, pp. 404--406 (2005)

15. Filho J.B., Martin. H.: A generalized context-based access control model for pervasive environments. In: the $2^{\text {nd }}$ International Workshop on Security and Privacy in GIS and LBS, pp. 12-21 (2009)

16. Zerkouk, M., M'hamed, A., Messabih, B., A User Profile Based Access Control Model and Architecture. In: I. J. Computer Networks \& Communications, Vol 5, $\mathrm{N}^{\circ} 1$, pp. 171-181 (2013) 